\title{
LA-UR-21-31065
}

Approved for public release; distribution is unlimited.

Title: $\quad$ Polymer Equations of State and Shock-Driven Decomposition

Author(s): $\quad$ Coe, Joshua Damon

Intended for: Invited talk at Sandia National Lab, Albuquerque

Issued: $\quad$ 2021-11-12 (rev.1) 
Disclaimer:

Los Alamos National Laboratory, an affirmative action/equal opportunity employer, is operated by Triad National Security, LLC for the National Nuclear Security Administration of U.S. Department of Energy under contract 89233218CNA000001. By approving this article, the publisher recognizes that the U.S. Government retains nonexclusive, royalty-free license to publish or reproduce the published form of this contribution, or to allow others to do so, for U.S. Government purposes. Los Alamos National Laboratory requests that the publisher identify this article as work performed under the auspices of the U.S. Department of Energy. Los Alamos National Laboratory strongly supports academic freedom and a researcher's right to publish; as an institution, however, the Laboratory does not endorse the viewpoint of a publication or guarantee its technical correctness. 


\section{Polymer Equations of State and Shock-Driven Decomposition}

Josh Coe Physics \& Chemistry of Materials (T-1) Los Alamos National Laboratory

November 9, 2021

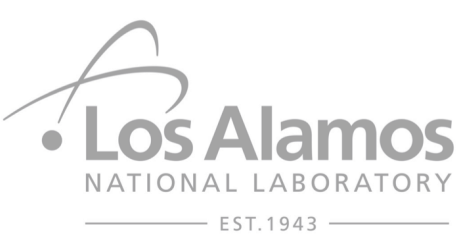




\section{Quick Overview}

- LANL started taking greater interest in polymer EOS in late 2000's

- Possible cause of poor simulation results

- Historically neglected

- Themes that have emerged from that work

- Polymers decompose chemically when driven at HE detonation pressures

- Porosity affects decomposition

- Decomposition affects flow

- Implications of $\uparrow$ for modeling 


\section{Polymer Hugoniots Contain Structure}

- Derivative discontinuities at $u_{p} \sim 3 \mathrm{~km} / \mathrm{s}$ ( $P \sim 25 \mathrm{GPa})$

- Middle line segment not at equilibrium

- Volume reduction in $P-V$

- Degree of reduction correlates qualitatively with chemical structure

\begin{tabular}{lcc}
\hline Material name & $P_{\text {threshold }}(\mathrm{GPa})$ & $\Delta V_{\mathbf{t r}} / V(\%)$ \\
\hline epoxy & 23.1 & 3.9 \\
PMMA & 26.2 & 3.4 \\
PTFE & 41.6 & 1.1 \\
PE (linear) & 24.7 & 0.4 \\
polycarbonate & 20.0 & 11.4 \\
phenolic & 23.2 & 6.7 \\
polysulfone & 18.5 & 12.9 \\
polyurethane & 21.7 & 7.3 \\
\hline
\end{tabular}

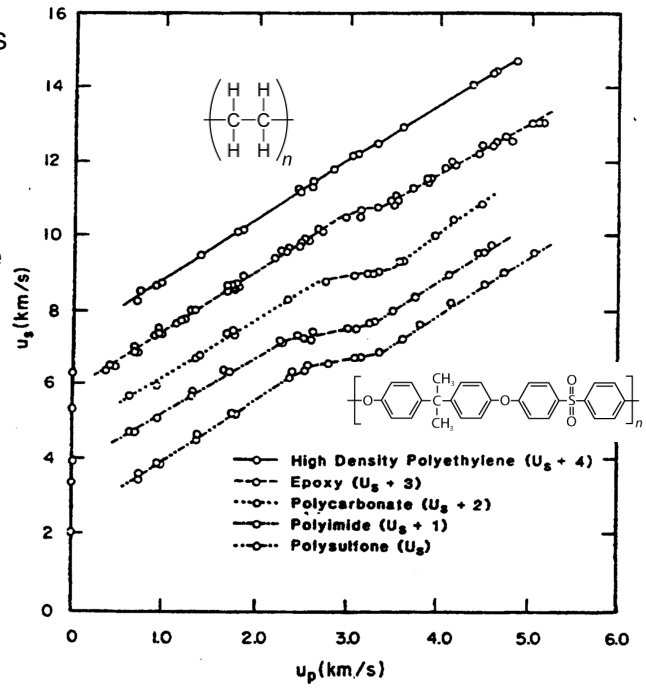




\section{Hugoniot Structure: Two Early Views}

- Phase transition (LANL, 1977)

- analogous to graphite $\rightarrow$ diamond

- "compression...is two-dimensional in nature" below the transition, "more typical of a three-dimensional solid" above

- Decomposition (LLNL, 1979)

- "..hydrocarbons at high pressure ( $\gtrsim 10 \mathrm{GPa})$ and high temperature $(\gtrsim 1000 \mathrm{~K})$ dissociate into carbon in the diamond phase and hydrogen in a condensed molecular phase"

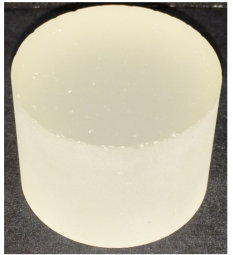

epoxy

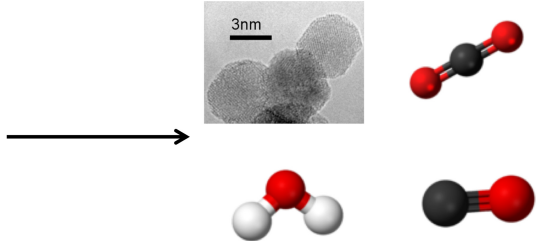

carbon $+\mathrm{H}_{2} \mathrm{O}+\mathrm{CO}+\mathrm{CO}_{2}+\ldots$ 


\section{Hugoniot Structure: Recovery Experiments}

- Experiments on polyethylene and Teflon

- Setup

- Single-shock, Mach compression

- Hermetically-sealed capsule

- Enabled recovery of soot and gases

- Mass spectrometry, XRD, TEM

- Polyethylene results

- Polymer recovered at $\sim 20 \mathrm{GPa}$

- Gases and soot recovered 28-40 GPa

- Gases were $>80 \% \mathrm{~mol} \mathrm{CH}_{4}$ and $\mathrm{H}_{2}$

- Soot was neither graphite nor diamond

$\Longrightarrow$ different material (with different EOS) when shocked above threshold

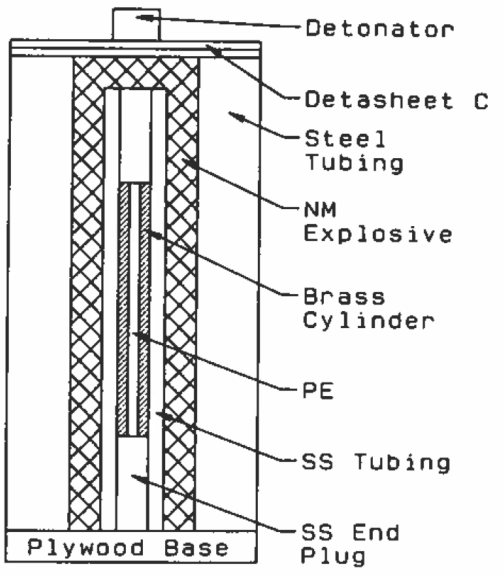




\section{Reactant EOS: SESAME Framework}

- Purely volumetric, no strength or viscoelasticity

- 3-part decomposition for free energy of each phase

$$
F(\rho, T)=\phi(\rho)+F_{\text {ion }}(\rho, T)+F_{\text {elec }}(\rho, T)
$$

- Minimize $F$ as function of mass fractions $\rightarrow$ equilibrium phase boundaries

- With regard to polymers:

- Cold curve extracted from fit to shock data

- This assumes Mie-Grüneisen form: $P(\rho, E)=P_{\text {ref }}(\rho)+\rho \Gamma(\rho)\left(E-E_{\text {ref }}(\rho)\right)$

- Ionic models are generalizations of Debye

- Polymer thermal response not well-described by single $\Theta$

- Thomas-Fermi-Dirac for electrons

- Electronic excitations not that important for $\rho_{\mathrm{H}} / \rho_{0} \lesssim 3$

- Typically one phase + liquid

- Liquid usually pretty hacky 


\section{Products EOS: Thermochemical Modeling}

- Decomposition products as mixture of fluids and bulk solids

- Each constituent has its own free energy model

- Fluids: spherical, pairwise interaction potential (EXP6) translated to free energy with perturbation theory

- Solids: SESAME model

- Mixture rule required (non-unique)

- Assume full thermodynamic (and thus, chemical) equilibrium

- Adjust concentrations until minimal free energy found and stoichiometry preserved
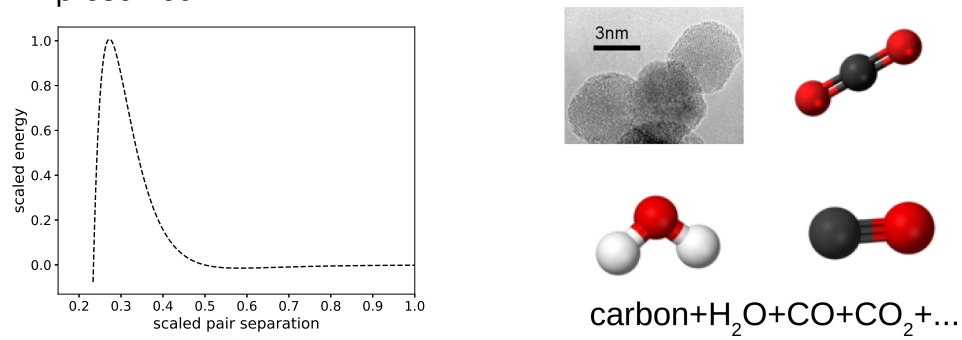


\section{Products EOS: Heat of Reaction}

- The origin of a products Hugoniot lies on a separate EOS surface

- Thermochemical EOS surface determined purely by stoichiometry

- Offset from the reactant surface is not

- closely related to heat of formation

- essential for capturing energy absorption or release

- We've done this in two ways:

- adjust to shock data

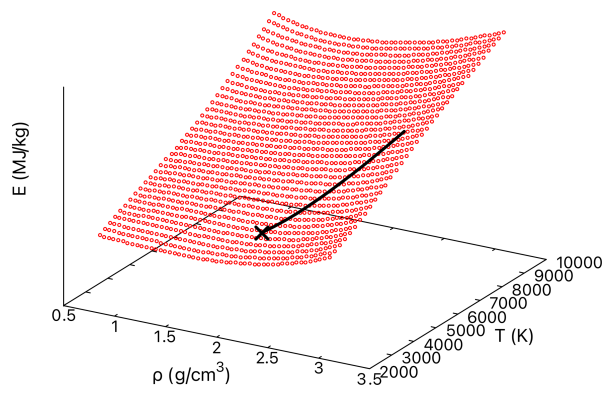

- calculate from heat of combustion 


\section{Products EOS: Heat of Reaction}

- The origin of a products Hugoniot lies on a separate EOS surface

- Thermochemical EOS surface determined purely by stoichiometry

- Offset from the reactant surface is not

- closely related to heat of formation

- essential for capturing energy absorption or release

- We've done this in two ways:

- adjust to shock data

- calculate from heat of combustion

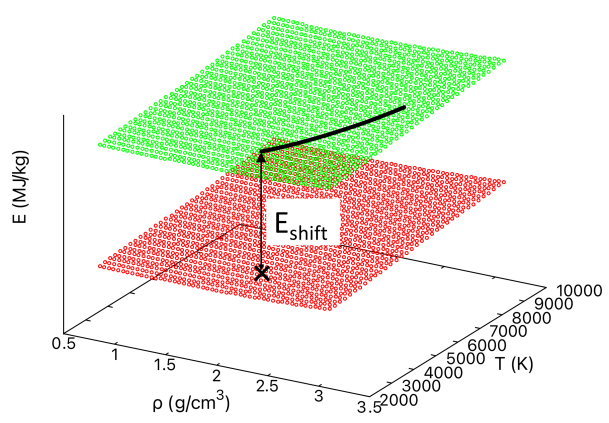




\section{Limitations of Thermochemical Modeling}

- Standard thermochemical tables are $T \sim 100^{\prime} s-10000 \mathrm{~K}, P \sim 0.01-150 \mathrm{GPa}$

- Limitations we understand:

- predefined constituent catalog

- potential form (mostly a problem for $\mathrm{H}_{2}$ )

- equilibrium assumption

- Stuff we're less sure about:

$-\mathcal{G}<0$ at high pressures

$-\Gamma<0$ at high and low $T$
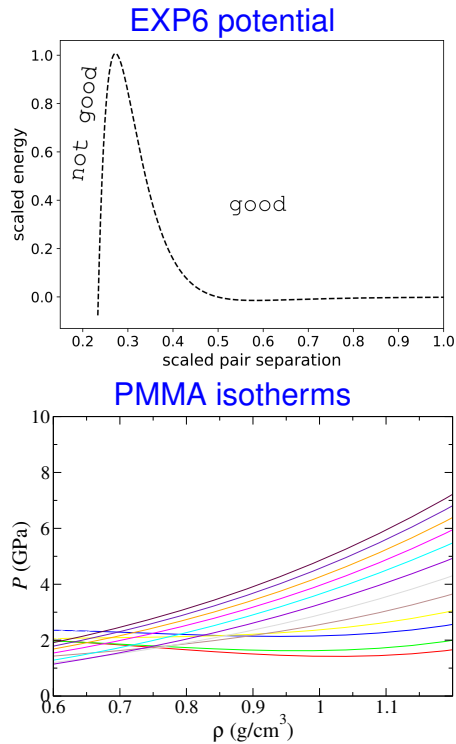


\section{PMMA: Shock}
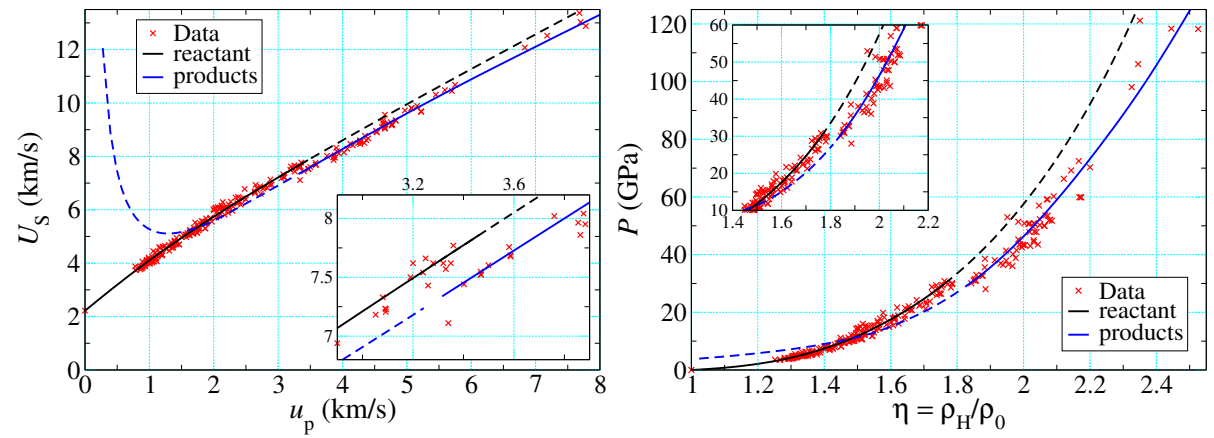

- Dashed segments indicated metastability (reactant) or physical impossibility (products)

- $E_{\text {shift }}$ from heat of combustion indistinguishable from optimized value 


\section{PMMA: Reshock and Release}
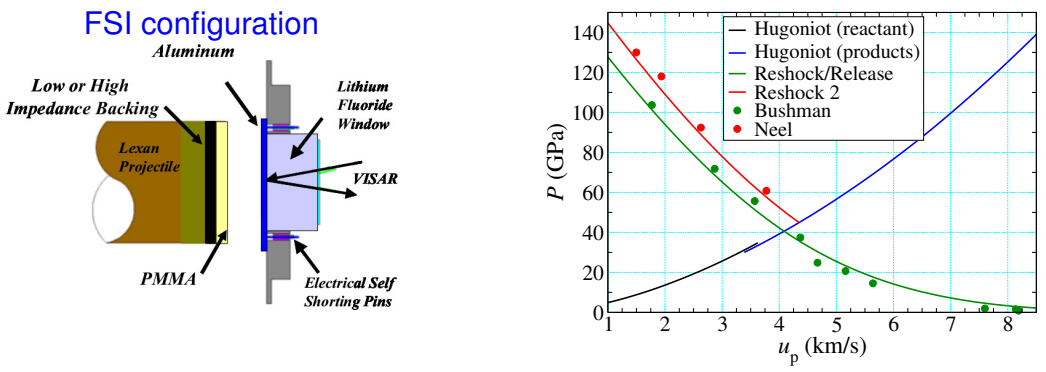

- SNL and Russian reshock/release experiments from common origin

- SNL: $P \approx 45 \mathrm{GPa}$

- Russian: $P \approx 41 \mathrm{GPa}$ 


\section{PMMA: Sounds Speeds at Pressure}

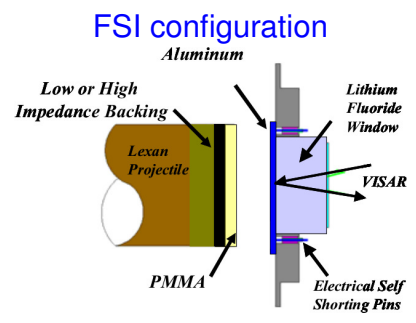

- $t_{3}$ is the observable, indicated by end of flat-top

- Metric is $\delta t_{3} /\left(t_{2}-t_{1}\right)$

- Errors in $C_{L}$ of $<5 \%$

- very similar results in PE
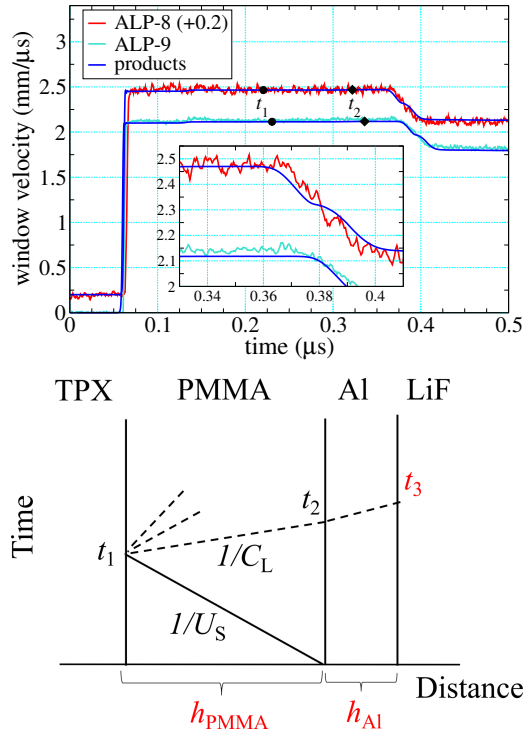


\section{PMMA: Product Compositions}
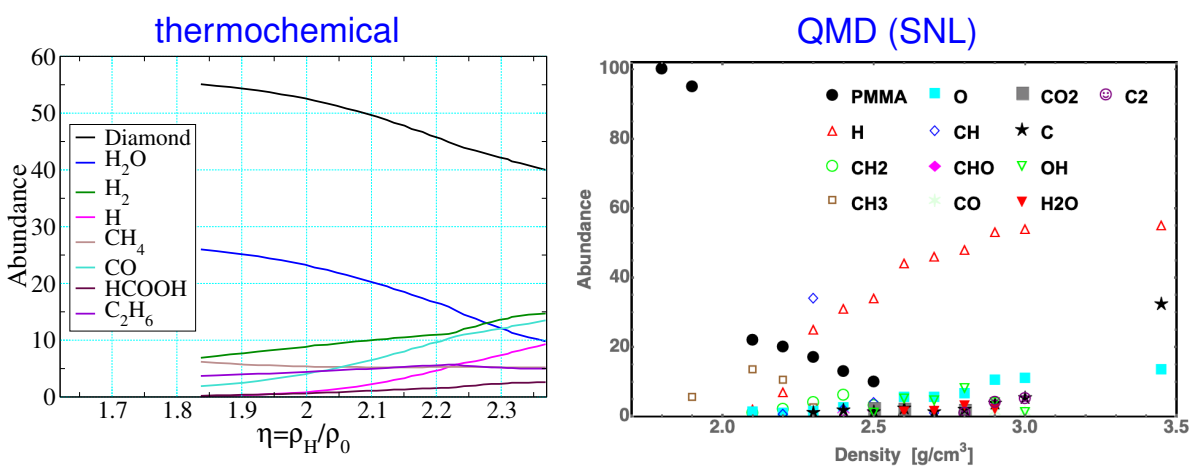

- Thermochemical products dominated by solid carbon and water

- Dominant change along the Hugoniot: $\mathrm{C}+\mathrm{H}_{2} \mathrm{O} \rightarrow \mathrm{CO}+\mathrm{H}_{2}$

- Thermochemical can't see reactant (no time), QMD can't see phase segregation (system size and timescale limitations)

- Oversimplified vs. uncertain relation to thermodynamics 


\section{Product Temperatures \& Reaction Thermicities}
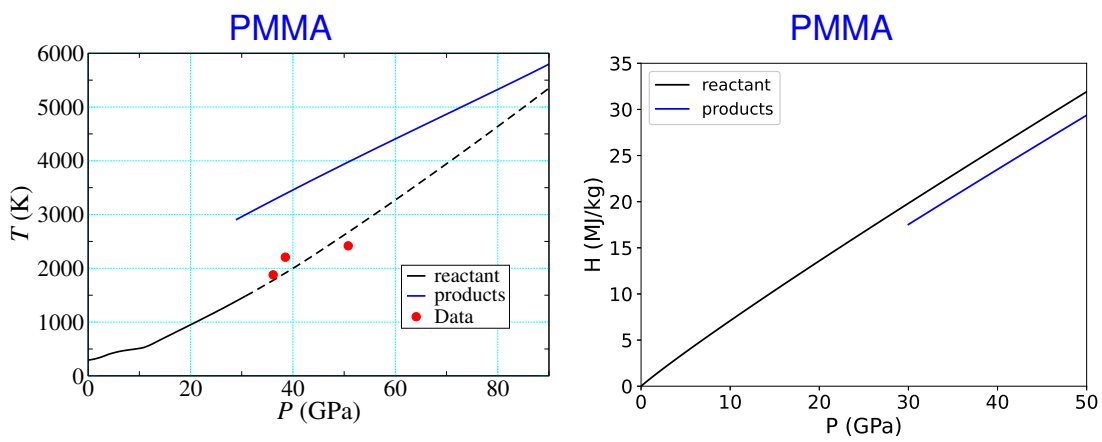

- Thermochemical modeling consistently predicts large temperature rises

- PMMA is only case in which we have actual data

- Temperatures are most poorly constrained thermochemical quantity

- Reaction at constant pressure is exothermic 


\section{Old way of capturing "chemistry"}

- Old EOS build structure into cold curve

- structure preserved in all isotherms until "washed out" by thermals

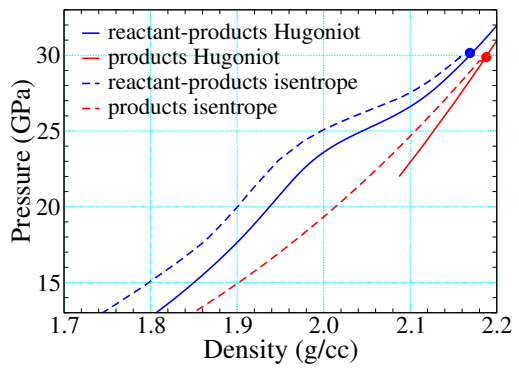

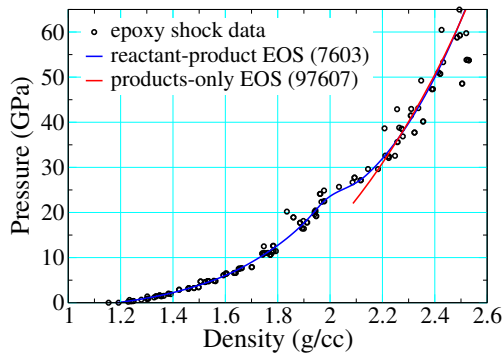

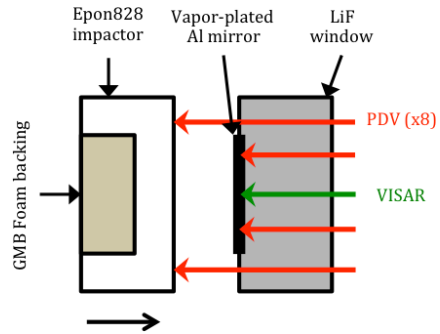




\section{Old way of capturing "chemistry"}

- Old EOS build structure into cold curve

- structure preserved in all isotherms until "washed out" by thermals

- produces multiwave structure upon release

- reversible phase transition rather than irreversible chemistry

- When might we care?
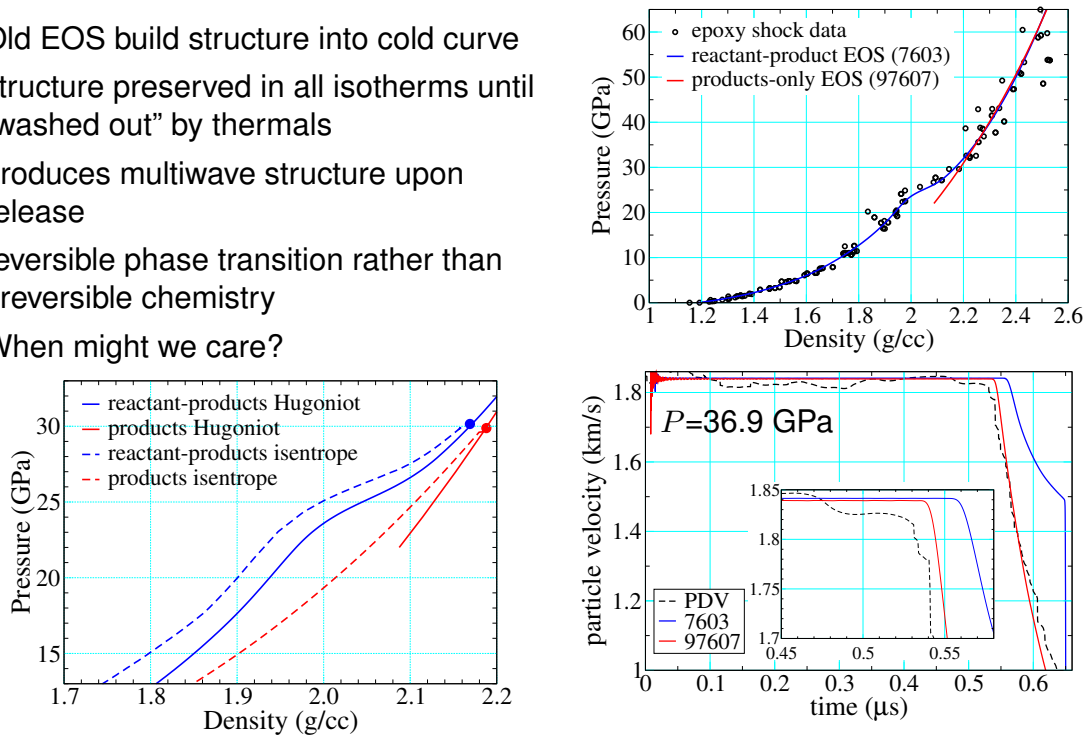


\section{Heterogeneous Materials are Horrible People}

\section{SX358}

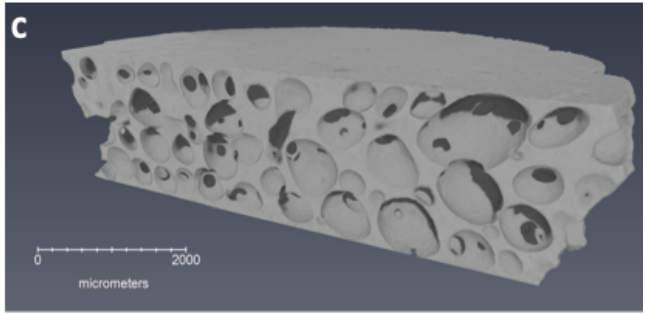

- Not always clear what you're probing

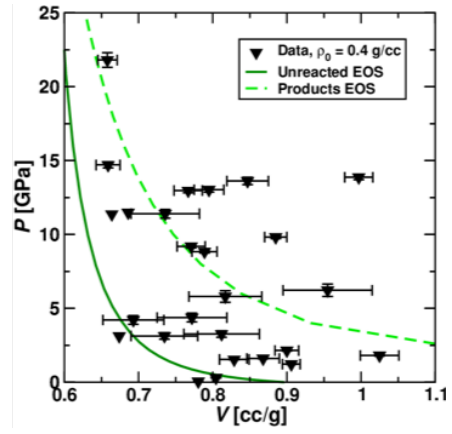

- Spot size of our standard PDV is roughly $450 \mu \mathrm{m}$

- Pore diameters span range $\mathcal{O}(10 \mu \mathrm{m}-1 \mathrm{~mm})$

- $U_{\mathrm{S}} \approx u_{\mathrm{p}}$, so $\sigma(\rho)$ large

- Shot-to-shot variability $>$ known sources of uncertainty

- We have the same problem with powders 


\section{Adjustments for Porosity}

polyurethane foams

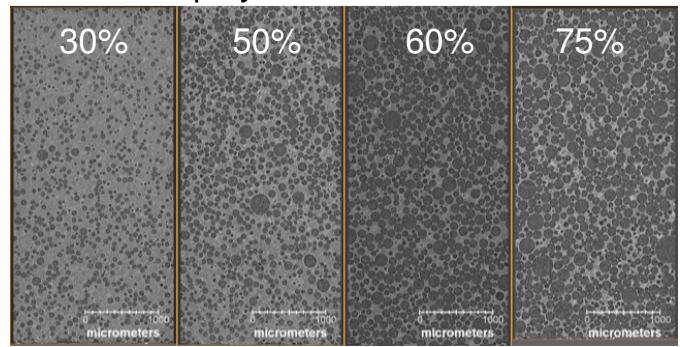

- Many polymers of interest to NNSA are porous, some highly

- Model reactant as SESAME $+P-\alpha$ porosity model

- Only porous parameter is crush pressure, $P_{c}$

- Still thermochemical modeling for products

- Vary $E_{\text {shift }}$ as $f\left(\rho_{0}\right)$, if necessary 


\section{Polyurethane Results}
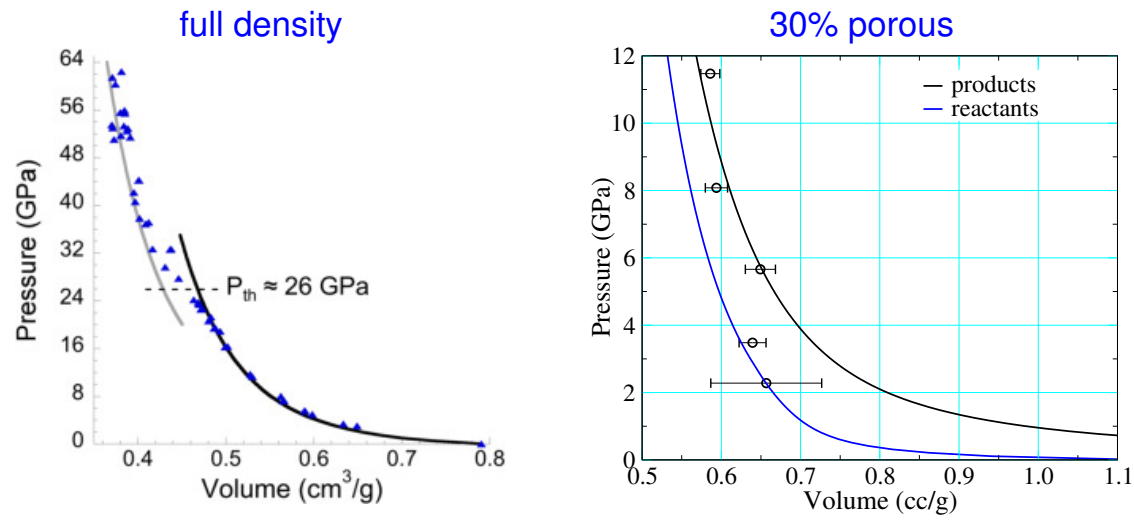

- Foam products expand relative to compressed reactant

- Reaction threshold drops dramatically as $f\left(\rho_{0}\right)$ 


\section{Polyurethane Results}
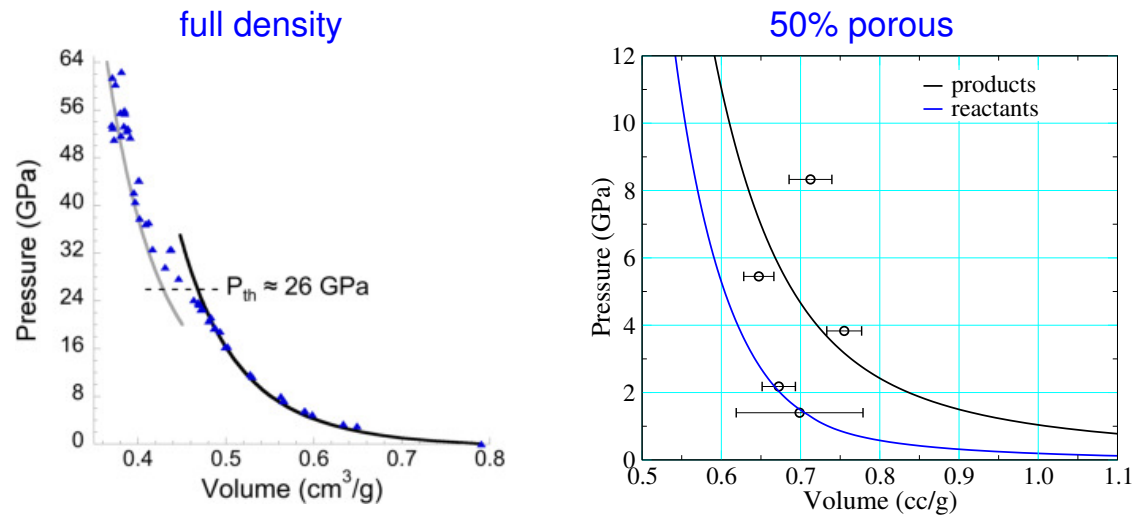

- Foam products expand relative to compressed reactant

- Reaction threshold drops dramatically as $f\left(\rho_{0}\right)$ 


\section{Polyurethane Results}
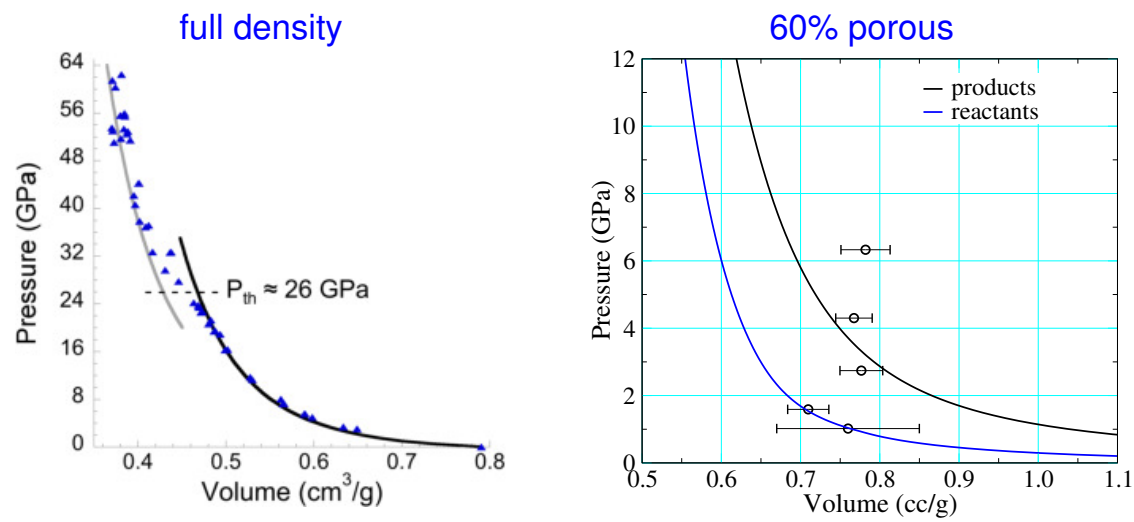

- Foam products expand relative to compressed reactant

- Reaction threshold drops dramatically as $f\left(\rho_{0}\right)$ 


\section{Polyurethane Results}
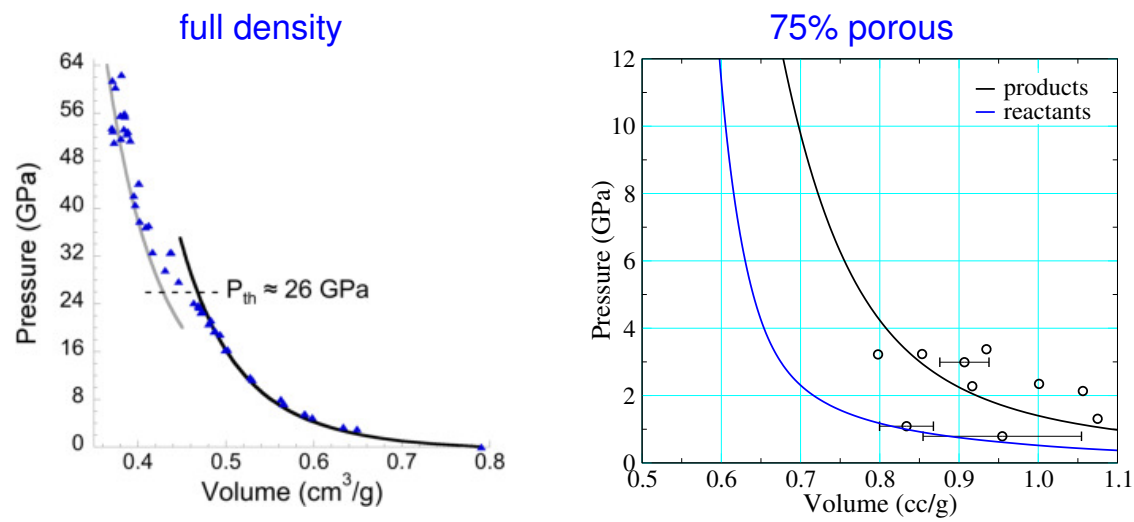

- Foam products expand relative to compressed reactant

- Reaction threshold drops dramatically as $f\left(\rho_{0}\right)$ 


\section{SX358 Results}
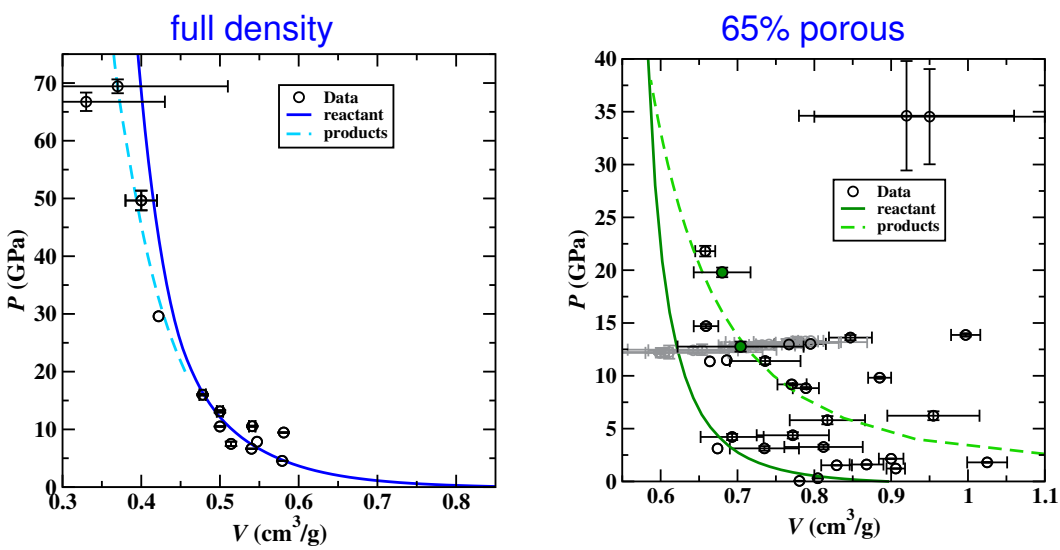

- Patterns consistent with polyurethane

- Uncertainties again: multislug, multi-PDV 


\section{Transition Thresholds}

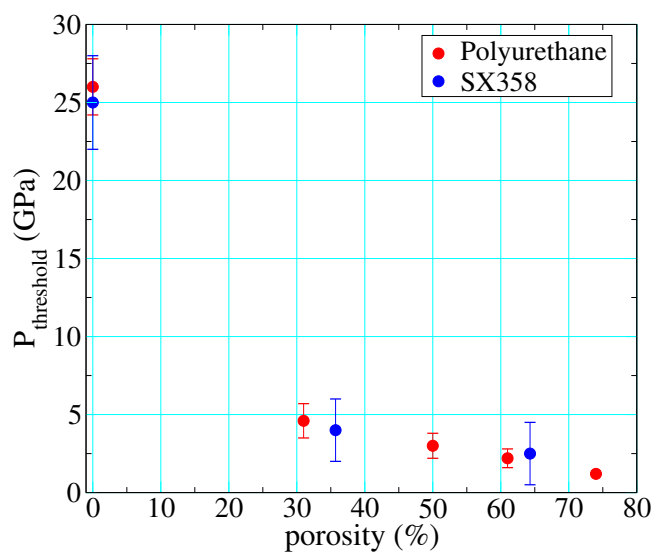

- Roughly exponential drop

- Strong dependence on timescale of experiment 


\section{Old Way of Treating Foams}

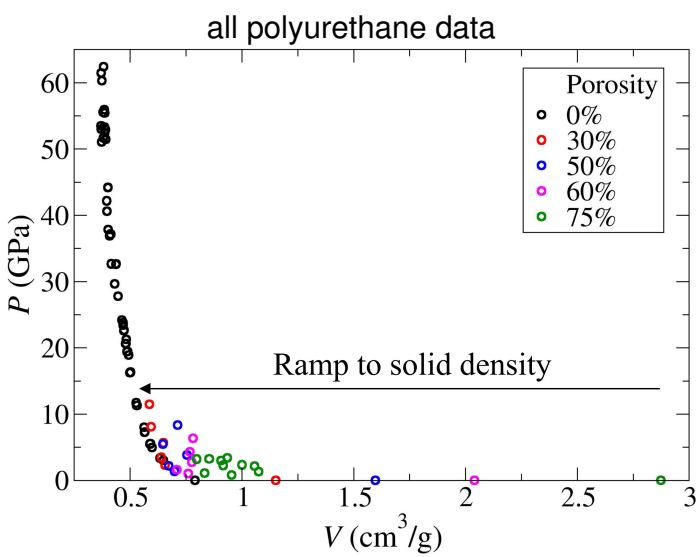

- Initialize at foam density, ramp to solid density Hugoniot

- Some error in density, energy, sound speed 


\section{Disentangling Non-Equilibrium Effects}

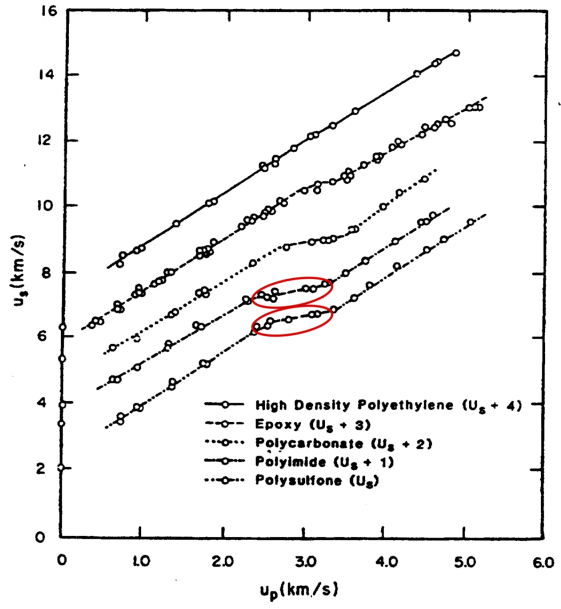

- 1 embedded gauge, 5 transmission shots on polyimide

- similar series on polysulfone 


\section{Measuring \& Simulating Reactive Wave Evolution In Situ}

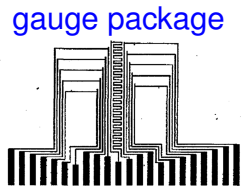

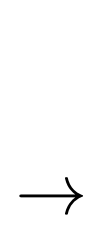

package in sample

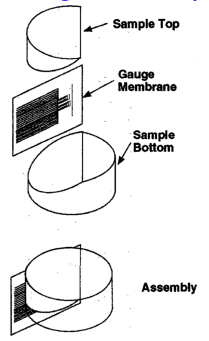

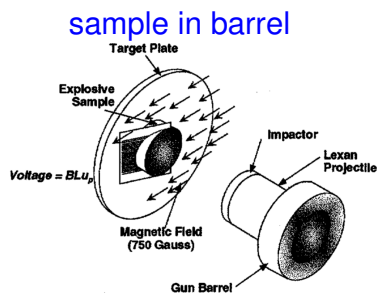

- Embedded electromagnetic gauges

- Advantage: minimizes measurement perturbation

- Disadvantage: limited to insulating samples and impactors

- Hydrodynamic simulation requires:

- Reactant and products EOS

- Rate model. We used Arrhenius: $\mathcal{R} \equiv \dot{\lambda}=(1-\lambda)^{n} \nu e^{-T_{a} / T}$

- Closure rule. We used pressure-temperature equilibrium. 


\section{In Situ Wave Proffles: Energetic Materials}
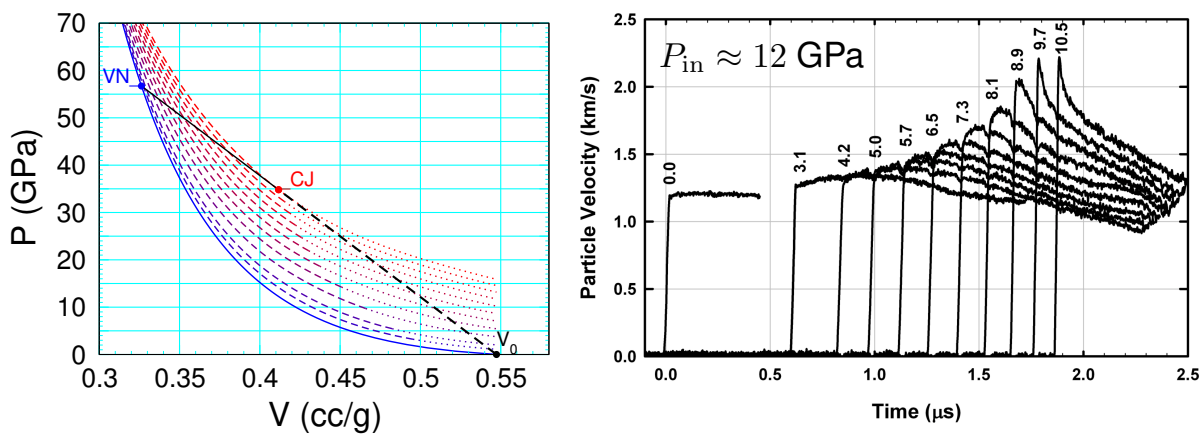

- ZND: inert shock followed by reaction zone to CJ state

- Reaction behind feeds the front, strengthening lead shock

- Reaction pushes unsteady $\rightarrow$ steady 


\section{In Situ Wave Profiles: Phenylacetylene}
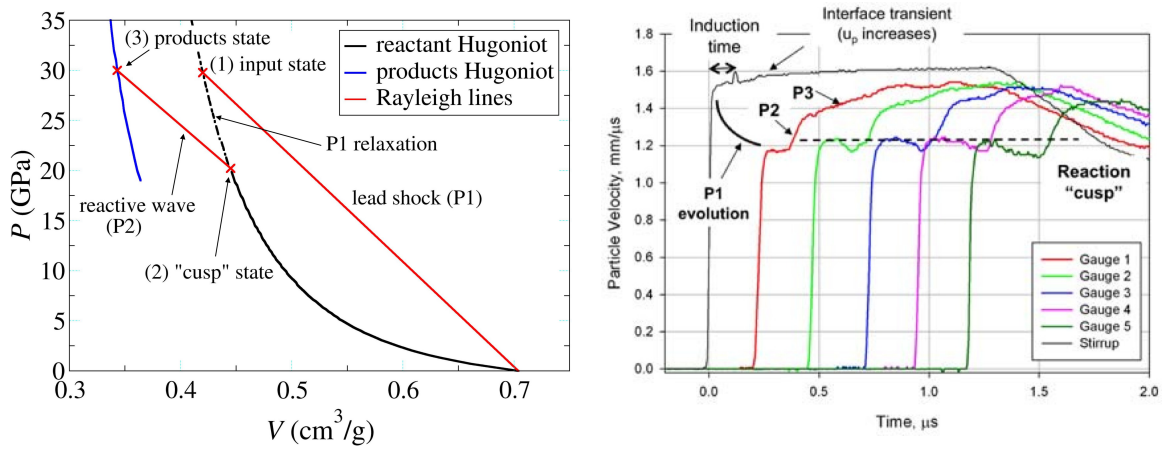

- Reaction behind the front weakens lead shock

- Initial (P1) wave decays, second (P2) wave carries to products

- Decay and rise times contain kinetic information 


\section{Rate Model Calibration}

- We used $1 /$ (adiabatic induction time) as a proxy for the rate

$$
\begin{gathered}
t_{\mathrm{ad}}\left(T_{0}\right)=\frac{T_{0}^{2}}{\nu T_{a}\left(T_{1}-T_{0}\right)} e^{\left(T_{a} / T_{0}\right)} \\
T_{0}=\text { reactant temperature } \\
T_{1}=\text { product temperature }
\end{gathered}
$$

- In our case, these are Hugoniot temperatures

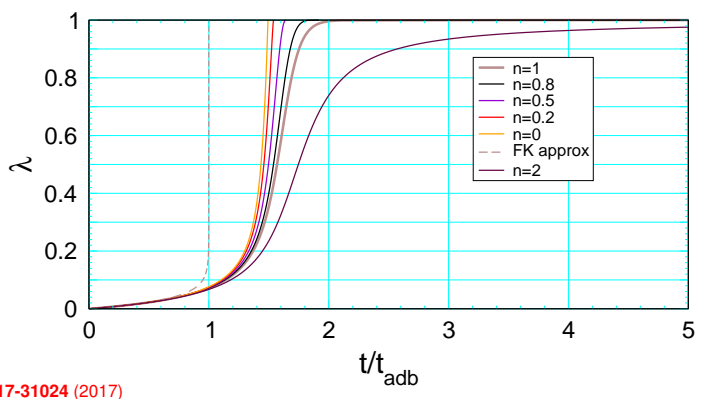




\section{Rate Model Calibration}

- Because $T_{0}=T_{0}\left(u_{p}\right)$, we'll consider $t_{\text {ad }}\left(u_{p}\right)$

- For a given pair of EOS:

- $T_{a}$ sets $u_{p}$ range

- $\nu$ shifts laterally
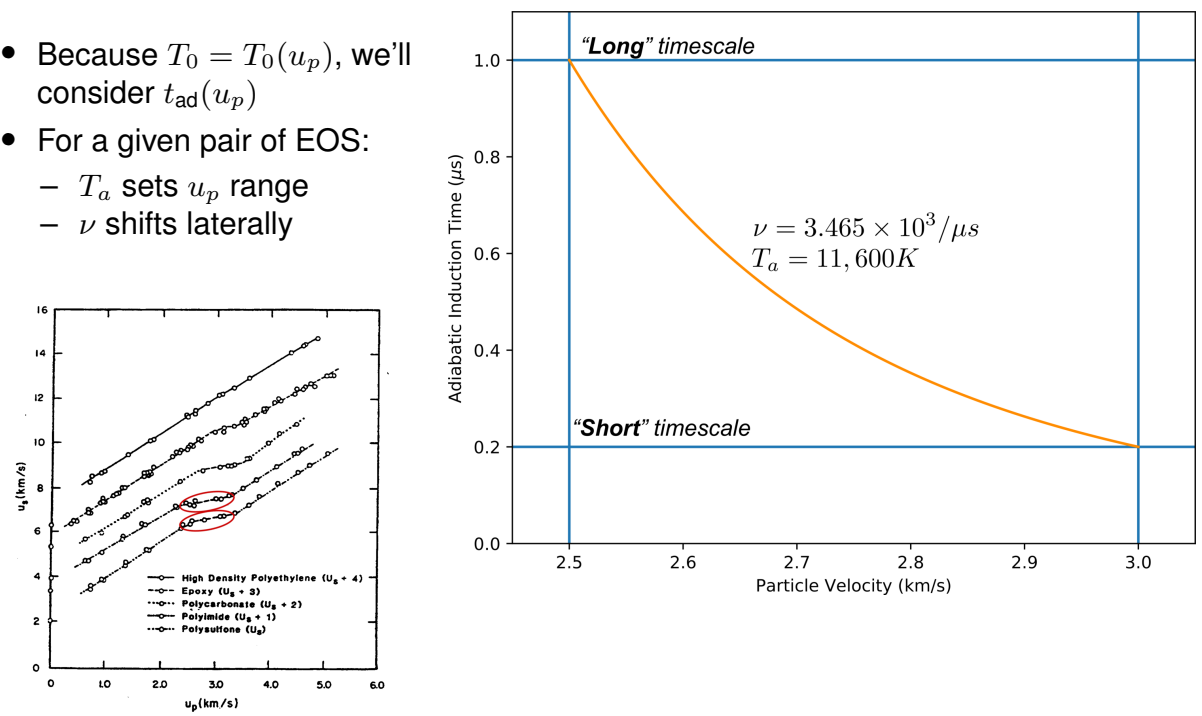


\section{Polyimide Shock Data}
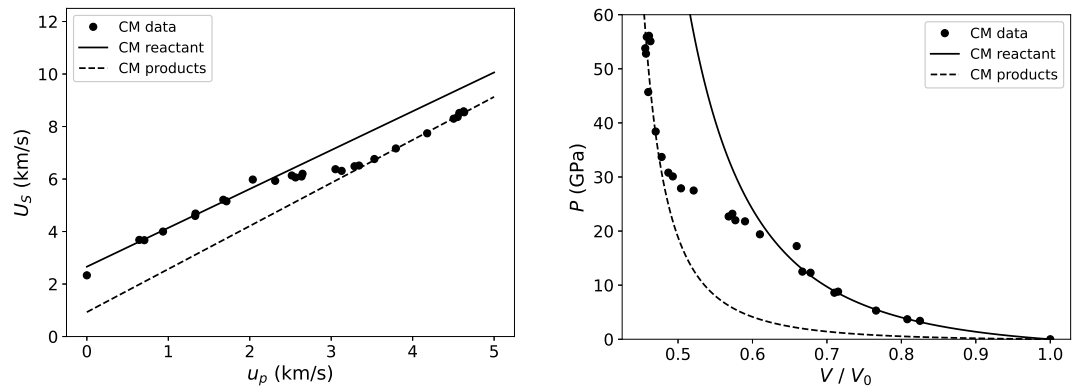

- CM reactant and products fits cross

- Is this partly due to their including reacting points in their reactant fit? 


\section{Polyimide: Embedded Gauge Results}
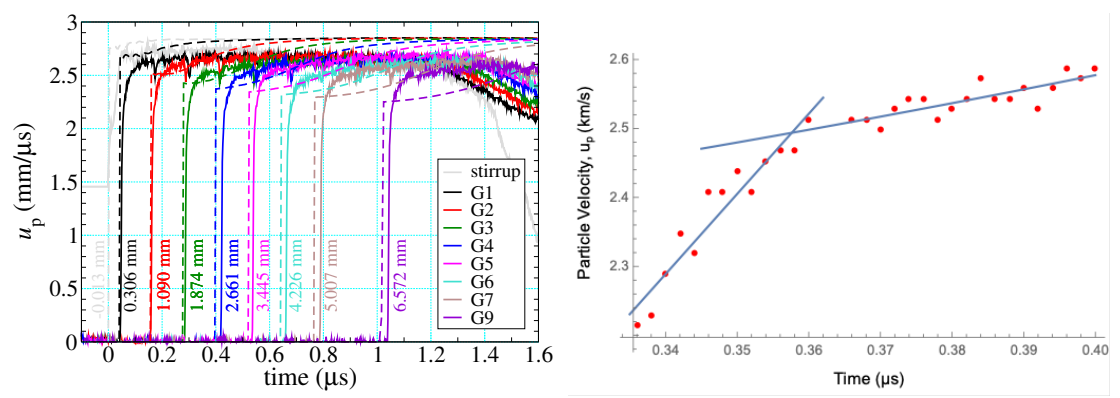

- Simulation: $50 \mu \mathrm{s}$ (Eulerian) mesh, $Z=830 \mu \mathrm{s}^{-1}, T_{a}=8560 \mathrm{~K}, n=2$

- Lagrangian tracers at gauge locations

- Experimental $u_{\mathrm{p}}$ analyzed similar to HE initiation data 


\section{Polyimide: Embedded Gauge Results}
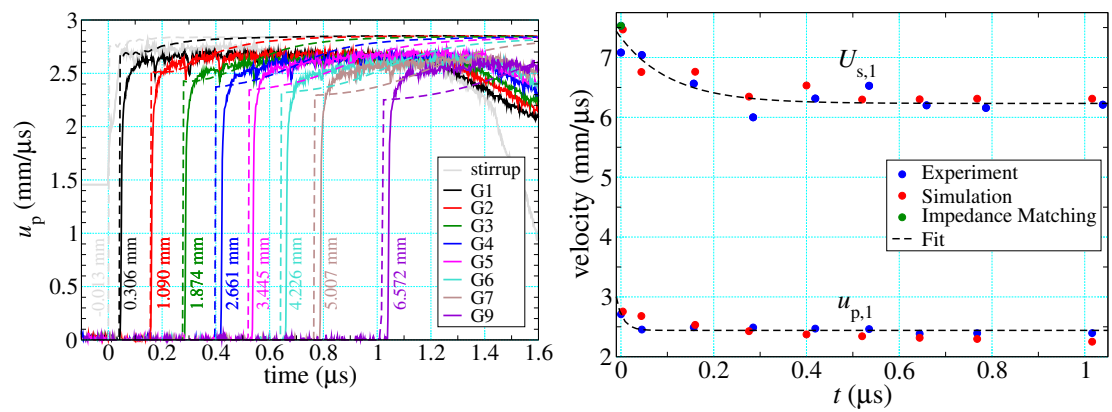

- Simulation: $50 \mu \mathrm{s}$ (Eulerian) mesh, $Z=830 \mu \mathrm{s}^{-1}, T_{a}=8560 \mathrm{~K}, n=2$

- Lagrangian tracers at gauge locations

- Experimental $u_{\mathrm{p}}$ analyzed similar to HE initiation data

- Attenuation of $\sim 15 \%$ in both $U_{\mathrm{S}}$ and $u_{\mathrm{p}}$, slightly large in simulation 


\section{Polyimide: Embedded Gauge Results}
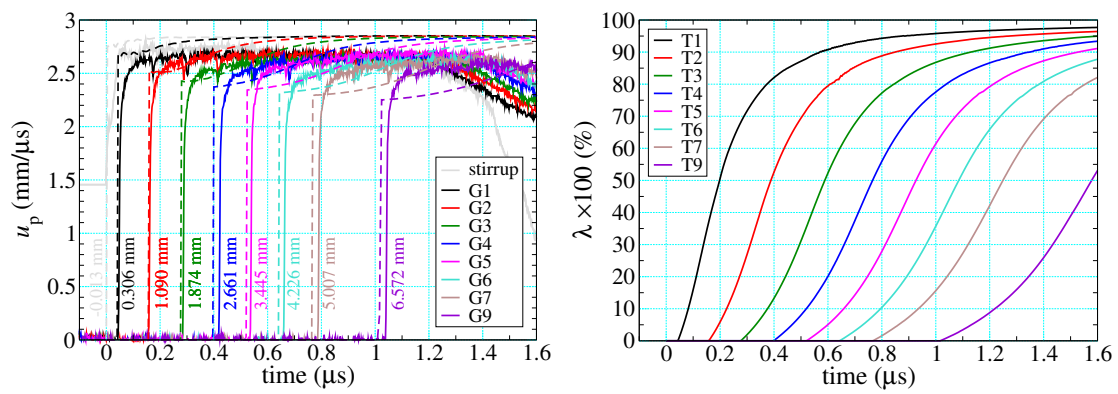

- Simulation: $50 \mu \mathrm{s}$ (Eulerian) mesh, $Z=830 \mu \mathrm{s}^{-1}, T_{a}=8560 \mathrm{~K}, n=2$

- Lagrangian tracers at gauge locations

- Experimental $u_{\mathrm{p}}$ analyzed similar to HE initiation data

- Attenuation of $\sim 15 \%$ in both $U_{\mathrm{S}}$ and $u_{\mathrm{p}}$, slightly large in simulation

- Data and simulations suggest sluggish reaction that doesn't proceed to completion $\Longrightarrow$ let's try a stronger input 


\section{Polyimide Shock Data}
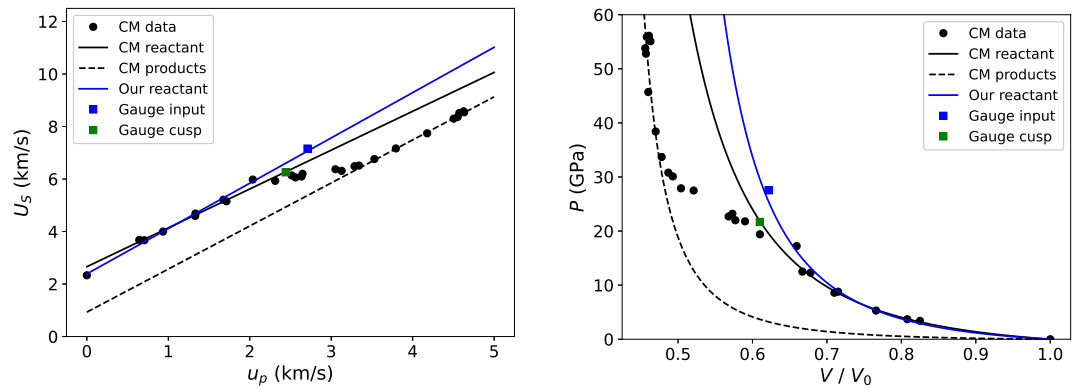

- CM reactant and products fits cross

- Is this partly due to their including reacting points in their reactant fit?

- Gauge results:

- Use input (blue square) to refit reactant (blue curve)

- Reactant and products no longer cross

- The cusp state (green square) lies on CM reactant (black line)

- Does P1 relaxation have a long "tail"? 


\section{Polyimide Transmission Analysis}

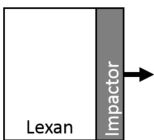

Projectile

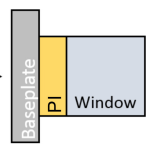

Target
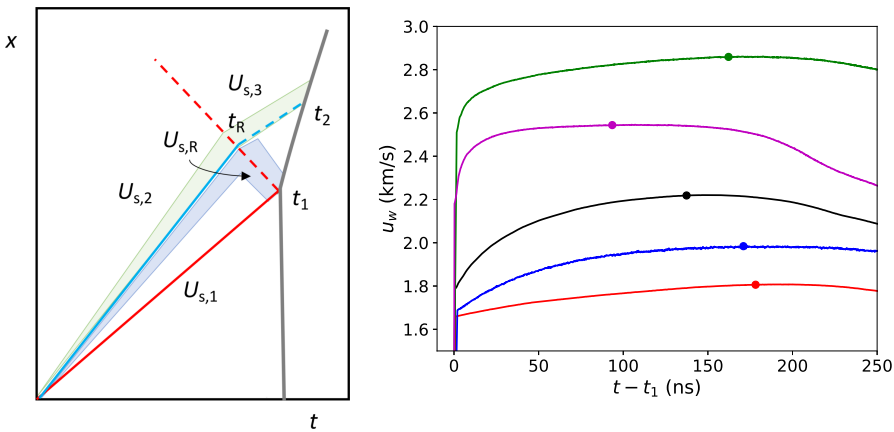

- Advantage: no restriction to non-conducting impactors

- Disadvantage: interaction with the window perturbs the flow

- The general problem is underdetermined - more unknowns than equations

- Similar configurations: Wackerle (1962), Ahrens (1968), Erskine (1992), McWilliams (2008)

- General theory: Courant \& Friedrichs, Zel'dovich, Forbes 


\section{Polyimide Transmission Analysis}

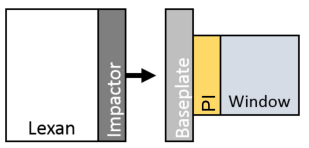

Projectile
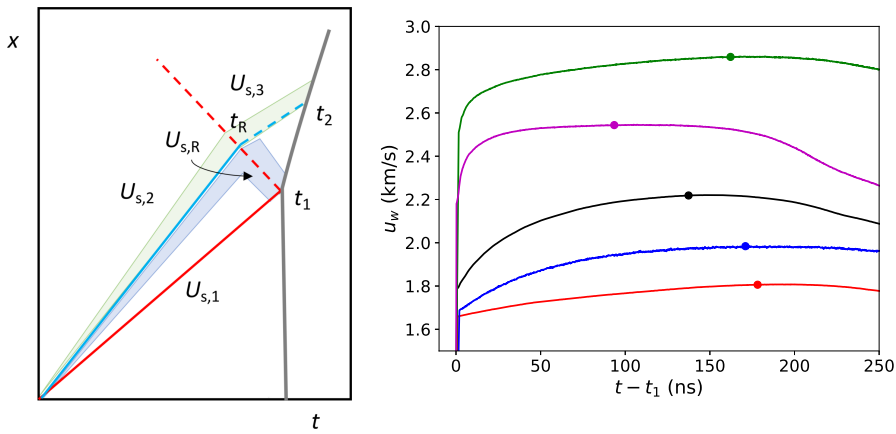

- Ignore wave-wave interaction $\Longrightarrow$ all about pinpointing $t_{2}$

- $P 2$ is "thickened" (Zel'dovich) - what are the constraints on $t_{2}$ ?

- $t_{1} \leq t_{2} \leq t\left(\max \left(u_{\mathrm{w}}\right)\right)$

- $t_{2}-t_{1}$ should decrease monotonically with $P_{\text {input }}$ 


\section{Polyimide Transmission Analysis}
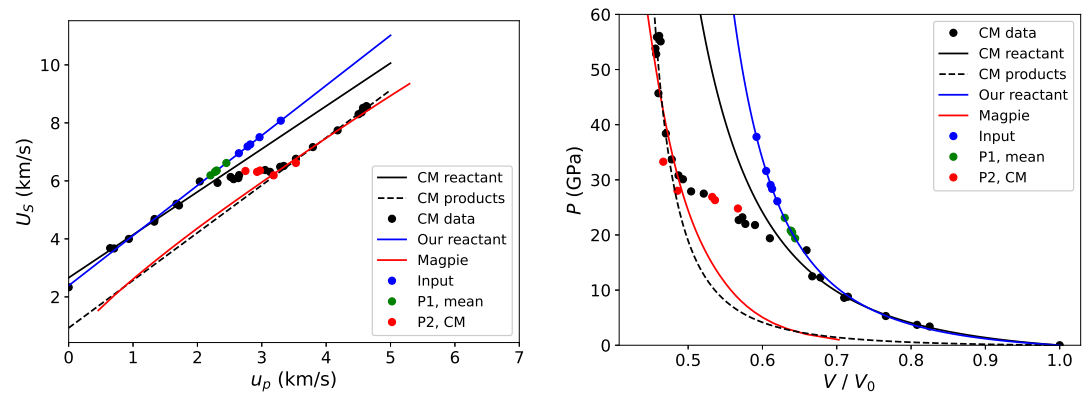

- Try the old way instead: $t_{2}=t_{1}$ (red points)

- Consistent with old results

- 2 of 5 points appear fully reacted

- So what is causing the structure in those profiles? 


\section{Polyimide Transmission Analysis}
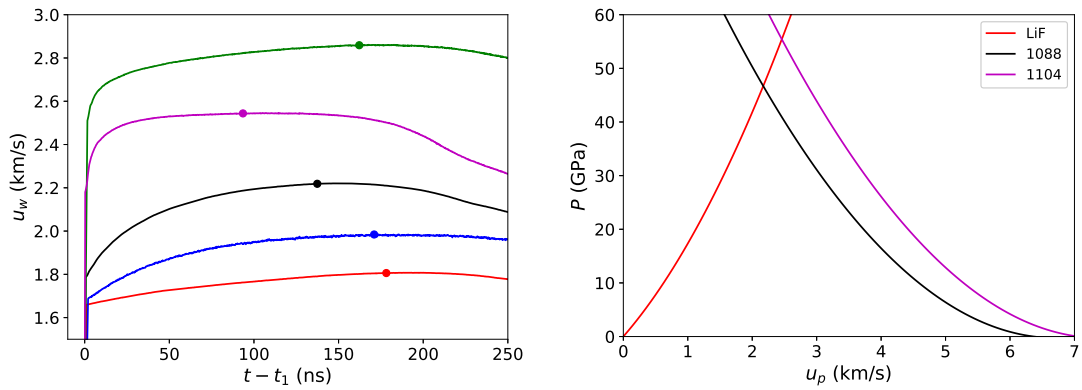

- Simplest reshock analysis: reflect old CM products fit and impedance match into LiF

- Yields window velocities correct to within a few \% (dashed, left panel)

- Reshocking one thickened wave yields...another thickened wave

- Shift in composition from equilibrium shock to equilibrium reshock 


\section{Polyimide Transmission Analysis}
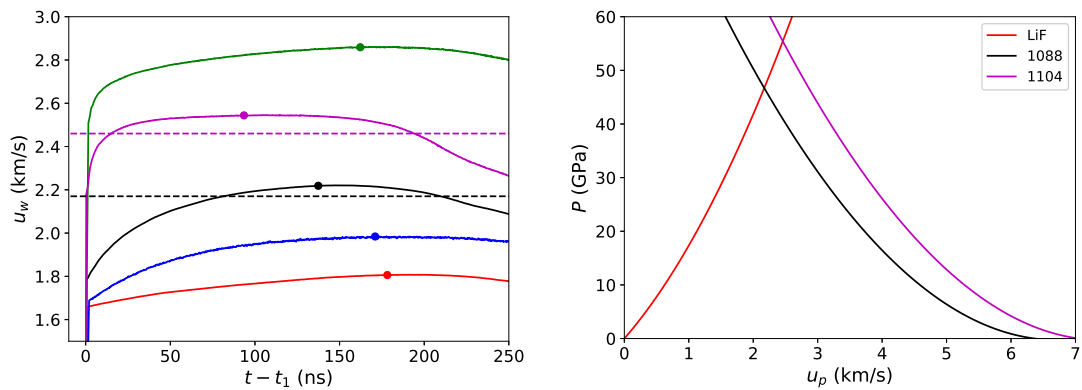

- Simplest reshock analysis: reflect old CM products fit and impedance match into LiF

- Yields window velocities correct to within a few \% (dashed, left panel)

- Reshocking one thickened wave yields...another thickened wave

- Shift in composition from equilibrium shock to equilibrium reshock 


\section{Polysulfone Transmission Experiments}

PDV at interface with window

increasing sample thickness $\rightarrow$

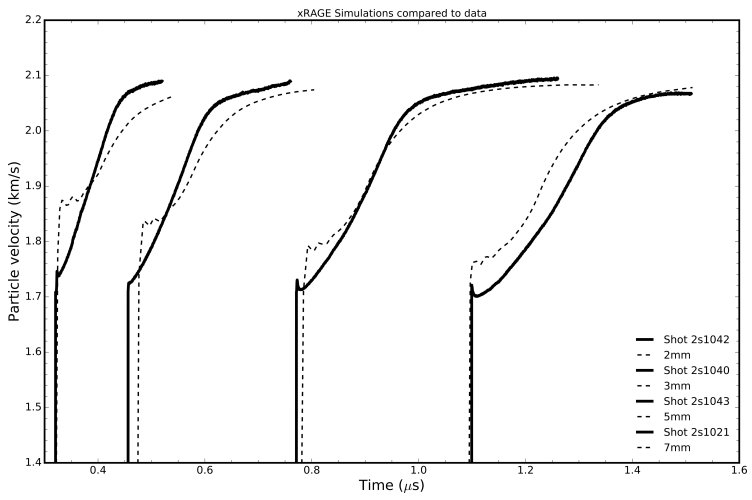

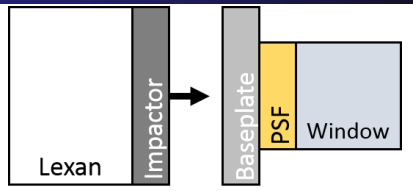

Projectile Target

Simulation

- underestimates P1 decay

- exaggerates P1 variation with thickness

- P2 slow when thin, fast when thick 


\section{Reactive Wave Profiles: Foams}

\section{PDV in polyurethane foams}
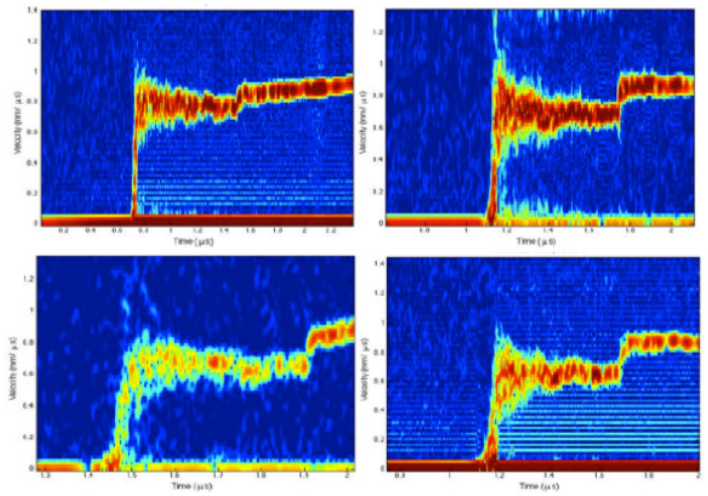

- Clockwise from upper left: 30\%, 50\%, 60\%, 75\% porous

- One wave observed

- PDV increasingly "bleached" due to high $T$ 


\section{Summary}

- Polymers decompose under shock loading

- $u_{p} \sim 3 \mathrm{~km} / \mathrm{s}, P \sim 25 \mathrm{GPa}$ at full density

- This is not a reversible phase transition..

- Details affected strongly by porosity

- Products expand upon reaction

- Thresholds drop dramatically

- Polymer decomposition produces non-trivial wave evolution

- Able to capture some qualitative features with simple rate model

- Qualitative features of transmission profiles resemble those of metals

- Unable to cleanly disentangle multiwave structure from interactions with window in transmission geometry 


\section{Acknowledgements, etc.}

- LANL: Dana Dattelbaum, Tinka Gammel, Rachel Huber, John Lang, Jeff Leiding, Katie Maerzke, Jeff Peterson, Chris Ticknor, Kirill Velizhanin

- SNL: Brittany Branch, Kyle Cochrane, Patricia Kalita, Chad McCoy, Josh Townsend

- \$: Science Campaign 2, ASC PEM

- References

- polyimide: in preparation, email jcoe@lanl.gov

- pmma: AIP Conf Proc 2272, 0700027 (2020), and in preparation

- polysulfone: JAP 127, 105902 (2020)

- Mie-Grüneisen form: https://arxiv.org/abs/2012.01169 (2020)

- review: Polymers 11, 493 (2019)

- polyethylene: JAP 126, 045902 (2019); AIP Conf Proc 1979, 030004 (2018)

- epoxy: AIP Conf Proc 1979, 090008 (2018)

- fiber-filled composites: JAP 116, 194308 (2014)

- polyurethane: JAP 115, 174908 (2014) 


\section{Extra Slides}




\section{Transmission Profiles in Metals}

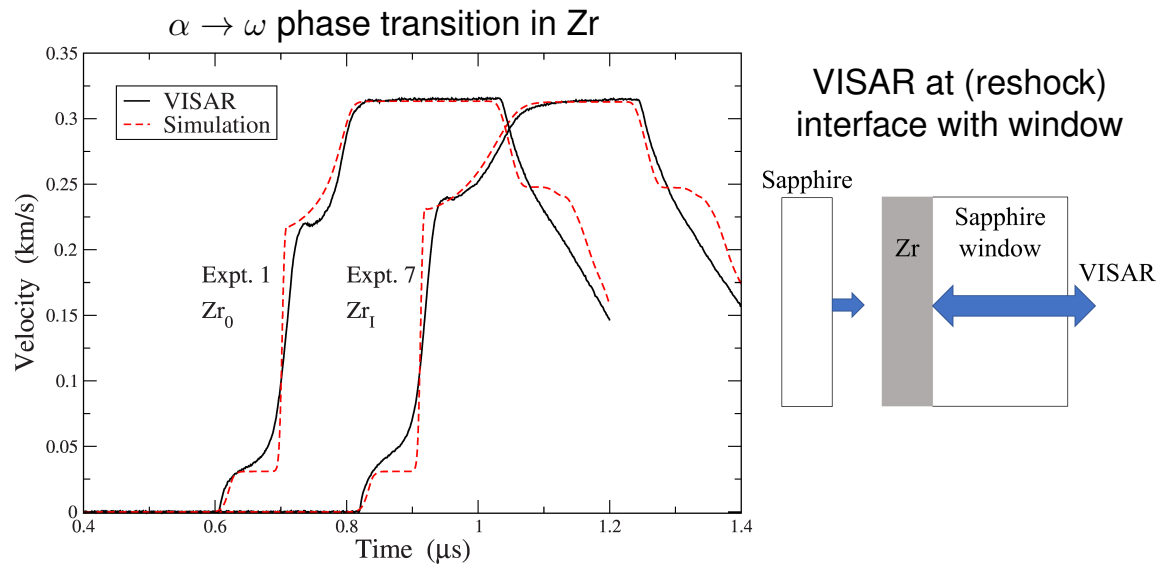




\section{Reactive Wave Profiles: Schematic}

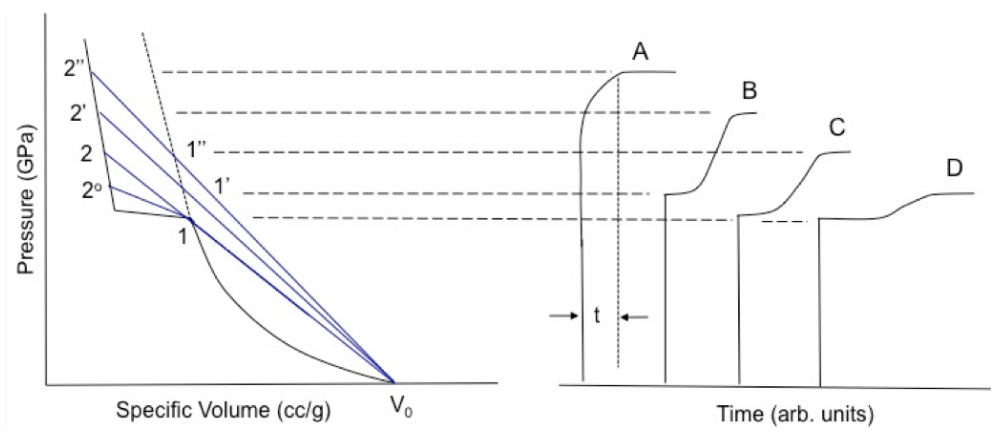

- Reaction behind weakens lead shock

- Waves separate rather than converge

- Initial (P1) wave decays, second (P2) wave carries to products

- Decay and rise times contain kinetic information 


\section{Detonation Criterion}

- In order to produce a self-sustaining wave, a material must have a positive thermicity coefficient, $\sigma$ :

$$
\begin{gathered}
\sigma=\left(\frac{\partial P}{\partial \lambda}\right)_{V, E}=\frac{\Delta V}{V}-\frac{\Gamma}{c^{2}} \Delta H \\
\lambda=\text { reaction progress variable } \\
\Gamma=\text { Grüneisen parameter } \\
c^{2}=(\partial P / \partial \rho)_{S, \lambda}=\text { frozen sound speed } \\
\Delta=(\partial / \partial \lambda)_{T, P}
\end{gathered}
$$

- Exothermicity $(\Delta H<0)$ isn't sufficient (or even necessary!) for detonation - "The importance of the volume term has often been overlooked..." 


\section{PMMA: Reactant Thermal}
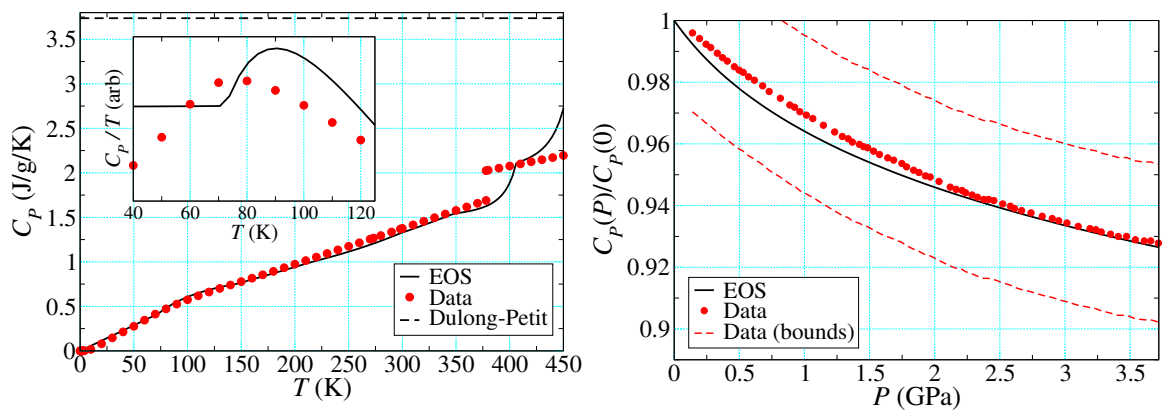

- $C_{P} \propto T$ at low $T$, never gets to classical limit

- We shove glass transitions and decomposition into melt

- Expansion surprisingly good given M-G constraint 


\section{PMMA: Reactant Thermal}
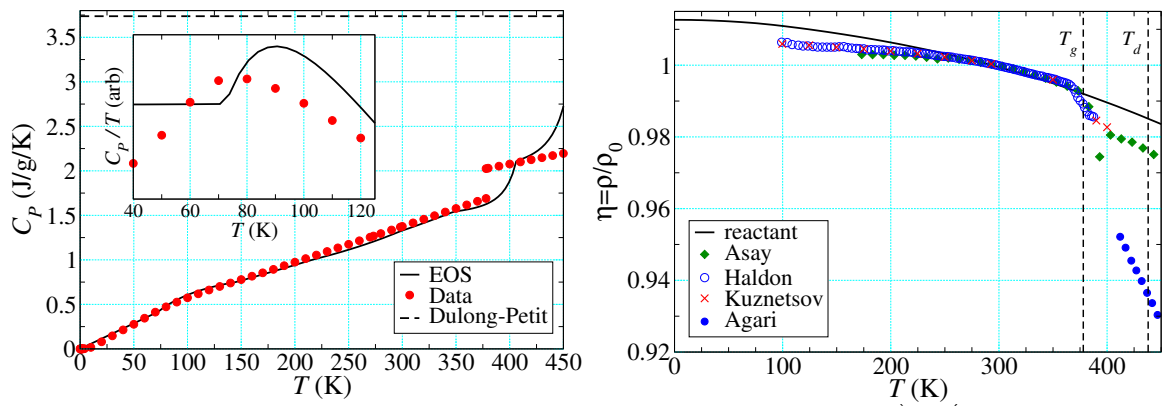

- $C_{P} \propto T$ at low $T$, never gets to classical limit

- We shove glass transitions and decomposition into melt

- Expansion surprisingly good given M-G constraint 


\section{Temperature Usually Increases}
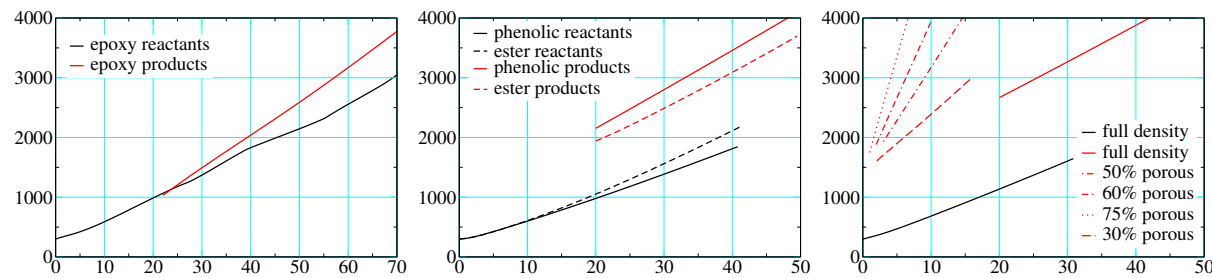

- In most cases we find $T>0$ upon decomposition

- Foam temperatures very high due to $P-V$ work

- High $T$ observable in "bleached" PDV signal 